\title{
Delayed ankle muscle reaction time in female amateur footballers after the first 15 min of a simulated prolonged football protocol
}

\author{
Daniel T. P. Fong ${ }^{1}$ D, Wing-Ching Leung ${ }^{2,3}$, Kam-Ming Mok ${ }^{2,4}$ and Patrick S. H. Yung ${ }^{2 *}$
}

\begin{abstract}
Purpose: Ankle sprain injury rate is reported to be higher towards the end of a football match. Muscle fatigue may contribute to the delayed muscle reaction and subsequent injury. This study investigated the ankle muscle reaction time during a simulated, prolonged football protocol.

Methods: Seven amateur female football players participated in a 105-min simulated, prolonged football protocol. An ankle muscle reaction test was conducted with a pair of ankle sprain simulators at a scheduled interval every 15-min. The reaction times of peroneus longus, tibialis anterior, and lateral gastrocnemius were collected using an electromyography system sampling at $1000 \mathrm{~Hz}$. Repeated measures one-way multivariate analysis of variance with post-hoc paired t-tests were conducted to evaluate if the reaction time at each time point significantly differed from baseline. Statistical significance was set at $p<0.05$ level.

Results: Reaction times started from 40.5-47.7 ms at baseline and increased to 48.6-55.7 ms at the end. Reaction times significantly increased in all muscles after the first 15 min except for the dominant lateral gastrocnemius. Increased reaction times were seen in the non-dominant limb after 60 min for tibialis anterior, after 75 min for peroneus longus, and after 90 min for the lateral gastrocnemius.

Conclusions: Delayed reaction time of the ankle muscles were found after the first $15 \mathrm{~min}$ and in the final $45 \mathrm{~min}$ of a simulated prolonged football protocol. Strategies for injury prevention should also focus on tackling the delayed ankle muscle reaction time in the acute phase (the first $15 \mathrm{~min}$ ), in addition to the latter minutes in the second half.
\end{abstract}

Level of evidence: Controlled laboratory study, Level V.

Keywords: Ankle sprain, Ligamentous sprain, Syndesmotic injury, Soccer, Sports medicine, Ankle injuries, Biomechanics

\footnotetext{
* Correspondence: patrickyung@cuhk.edu.hk

${ }^{2}$ Department of Orthopaedics and Traumatology, Prince of Wales Hospital, Faculty of Medicine, The Chinese University of Hong Kong, Hong Kong,

China

Full list of author information is available at the end of the article
}

(๑) The Author(s). 2020 Open Access This article is licensed under a Creative Commons Attribution 4.0 International License, which permits use, sharing, adaptation, distribution and reproduction in any medium or format, as long as you give appropriate credit to the original author(s) and the source, provide a link to the Creative Commons licence, and indicate if changes were made. The images or other third party material in this article are included in the article's Creative Commons licence, unless indicated otherwise in a credit line to the material. If material is not included in the article's Creative Commons licence and your intended use is not permitted by statutory regulation or exceeds the permitted use, you will need to obtain permission directly from the copyright holder. To view a copy of this licence, visit http://creativecommons.org/licenses/by/4.0/. 


\section{Background}

World-wide participation rates in women's football increased dramatically from 2010 to 2020 and are projected to double by 2026 [1]. Historically, injury incidence has been high in women's football [2] and remains so today [3]. It is expected that female footballrelated injuries and longer-term wellbeing will become a significant topic in sports medicine as evidenced by several recent publications [4-6]. A recent systematic review also suggested that recreational football practice is medicine - it is beneficial for cardiovascular and bone health, body composition, type 2 diabetes, and prostate cancer [7]. Previous research has demonstrated that greater attention to injury prevention can mitigate injury risk, allowing athletes to fully benefit from football practice. In this scenario, additional gaps need to be fulfilled regarding excessive ankle inversion caused by incorrect landing posture [8], muscle fatigue during prolonged football exercise [9] and delayed peroneal muscle reaction time [10]. If we can understand the cause of injuries better and introduce appropriate injury prevention strategies, we can ensure that footballers enjoy the health benefits of the game rather than suffer negative consequences.

Systematic reviews $[11,12]$ and also some recent epidemiology studies on female footballers $[13,14]$ suggest that ankle sprain is the most common single type of injury in football. In women's football, an epidemiological study on German premier league players reported that ankle sprains accounted for $35.4 \%$ of all injuries incurred in one season [15]. The commonly suggested aetiology of inversion type ankle sprain injury is incorrect positioning of the foot when transitioning from a nonweight-bearing to weight-bearing situation. An aberrant plantar flexed position of the ankle joint [8] increases the torque around the sub-talar joint thus increasing the likelihood of an inversion sprain. Peak inversion during ankle sprain has been reported to reach between 48 and 78 degrees [16, 17], with other studies reporting aberrant inversion being a common finding in chronic ankle instability $[18,19]$. The delayed reaction time of the peroneal muscles at the lateral aspect of the ankle, which is usually $60-90 \mathrm{~ms}$, is perhaps too slow to catch up with a quick inversion type ankle sprain motion that happens within $50 \mathrm{~ms}$ [16]. Previous studies also suggested that in real football game situations, ankle sprains are more likely to occur during the latter minutes of the first half and during the second half [20]. A recent review suggested that muscle fatigue by the end of a prolonged football game may contribute to the decline in the capability of muscle to generate force, the fall in physical performance, and reduced central drive from the nervous system. All these may predispose the athlete to ankle sprain injury [9]. Another recent study also suggested that football simulated fatigue resulted in balance impairment, which may be a contributory factor toward increased injury risk in the latter part of football games [21].

The presence of fatigue and chronic ankle instability together could disrupt dynamic postural control of the ankle joint [22], making the ankle joint more vulnerable to sustain an ankle sprain injury. To simulate the conditions experienced with prolonged football exercise, there have been numerous studies that have fatigued the ankle evertor muscles via repetitive exercise performed on an isokinetic dynamometer [23]. However, these protocols may not truly represent the physical demands of gameplay and the multi-directional nature of the sport [24]. We aimed to study the ankle evertor muscle fatigue in a realistic simulation of a football match to better understand the aetiology of ankle sprain injury. The purpose of this study was to investigate the ankle muscle reaction in a group of female amateur football players during a functional prolonged football exercise protocol. The hypothesis was that ankle evertor muscle fatigue will cause an increase in the reaction time of the lower leg muscles to an ankle inversion perturbation.

\section{Methods}

\section{Participants}

Seven female football players from the local female amateur football league participated in this study. The exclusion criteria included ankle instability, balance problems, serious foot problems, or lower limb or back fracture within 1 year, as evaluated by an orthopaedic specialist. The subjects were instructed not to perform any vigorous exercise $24 \mathrm{~h}$ before testing. Informed consent was obtained from each subject. The Joint Chinese University of Hong Kong - New Territories East Cluster Clinical Research Ethics Committee approved the study (CRE-2010.587).

\section{Sample size calculation}

Sample size estimation was done in G*Power software (Germany), based on a previous study, which reported that the reaction time of peroneus longus to be $68.8 \pm$ $4.5 \mathrm{~ms}$ in the stable ankle, and $84.5 \pm 4.6 \mathrm{~ms}$ in the unstable ankle without tape [25]. By setting the level of significance to 0.05 and the statistical power to 0.80 in a two-tailed test on two independent groups, the estimated required sample size was calculated to be 6 .

\section{Experimental design}

Each subject participated in a protocol (Fig. 1) modified from the Loughborough Intermittent Shuttle Test [26]. Before the exercise protocol, a standardized warm-up consisting of jogging and stretching was performed for $15 \mathrm{~min}$. All subjects were equipped with a heart rate 


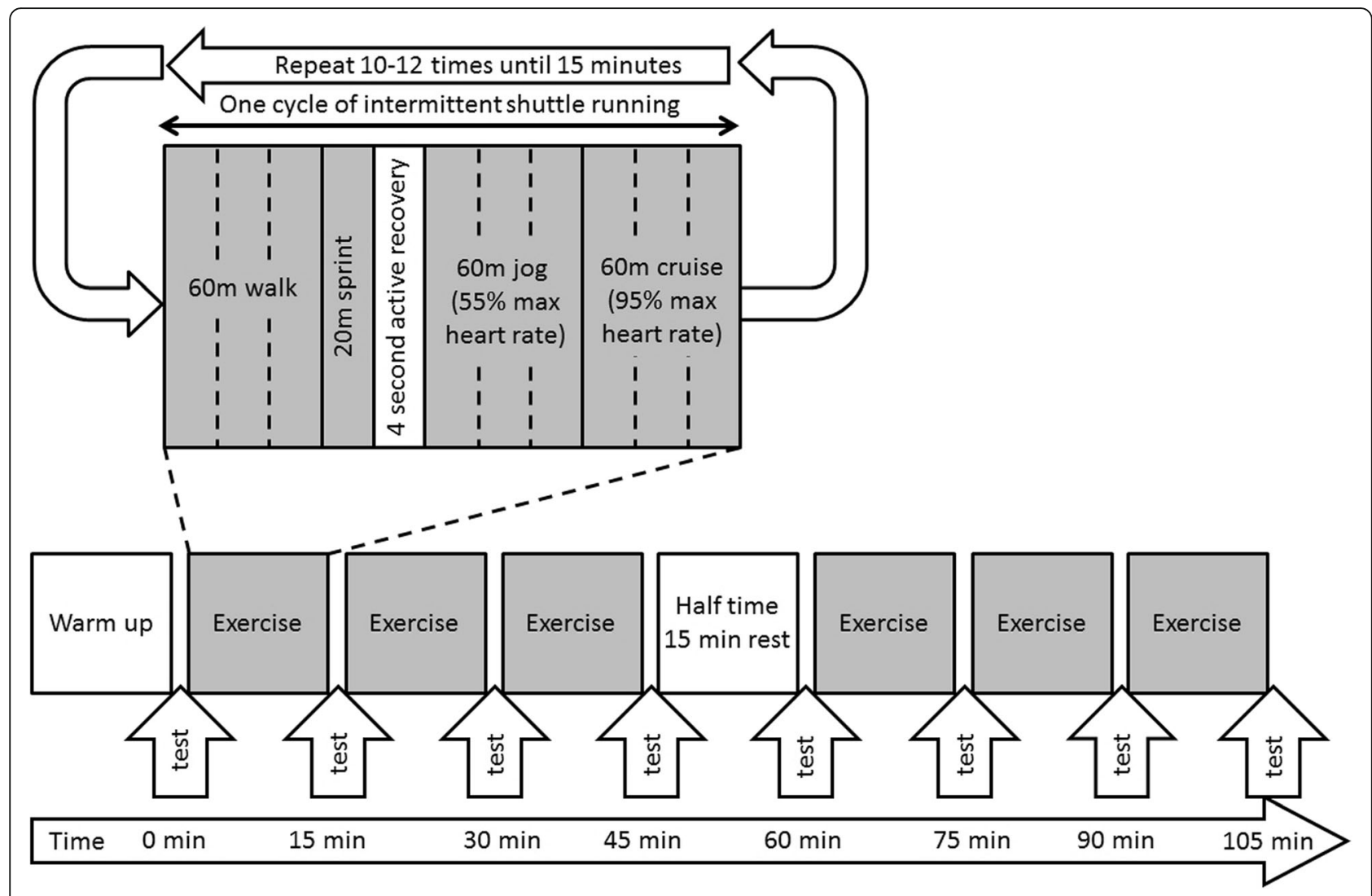

Fig. 1 The prolonged simulated football protocol used in this study

monitor (Polar Sport tester, Polar Electro Oy, Kempele, Finland), and their maximum heart rate was estimated by 220 minus their age. The protocol contained two sessions of 45-min continuous intermittent shuttle running exercises on hard ground, with a 15-min rest in between. The participants were required to run between two cones $20 \mathrm{~m}$ apart, at speeds related to the estimated individual heart rate values. Each cycle of the intermittent shuttle running exercise consisted of $60 \mathrm{~m}$ walking, $20 \mathrm{~m}$ sprinting, 4-s recovery walks, $60 \mathrm{~m}$ jogging at $55 \%$ maximum heart rate, and $60 \mathrm{~m}$ cruising at $95 \%$ maximum heart rate. A research assistant monitored the heart rate and gave verbal instructions to the subject to run faster or slower to achieve the targeted heart rate. The cycle was repeated for around 10-12 times until 15 min were reached, at which point a short interval was taken to conduct an ankle muscle reaction test before the next cycle began.

The ankle muscle reaction test was conducted at every 15-min interval, i.e. at $0,15,30,45,60,75,90$, and 105 min. The subject stepped on a pair of ankle sprain simulators which elicited an ankle muscle reaction to resist sudden simulated ankle inversion perturbation [27]. Three pairs of surface electrodes (3 M Red Dot Electrodes, diameter $=6 \mathrm{~cm}$, inter-electrode distance $=2 \mathrm{~cm}$,
USA) were attached on the skin surface of the peroneus longus, tibialis anterior and lateral gastrocnemius muscles. The electromyography (EMG) signals were collected via an EMG system with $1000 \mathrm{~Hz}$ sampling rate (TeleMyo 2400 T G2, Noraxon Inc., USA), as shown in Fig. 2. The muscle positions were identified by a method suggested in an EMG manual [28]. A reference electrode was attached to the lateral femoral condyle. Subjects were instructed to stand on the platforms as they usually do on hard ground, with weight equally distributed on both limbs and without additional lower limb muscle contraction as monitored by the EMG system. Sudden simulated ankle inversion perturbations were introduced to the dominant and non-dominant limb in random sequence, until three trials on each limb were collected. Dominant limb was defined as the preferred limb to kick a ball as verbally reported by the participant. Two electrogoniometers (SG110, Biometrics Ltd., UK) were attached to the posterior shanks and heels to identify the time of the start of inversion as when the inversion angle changed by 0.1 degrees. The time of onset of the EMG signal at each muscle was determined by a sudden signal increase which exceeded $5 \%$ of the maximum signal value of each channel. The time between the start of ankle inversion and the onset of EMG signal was the 


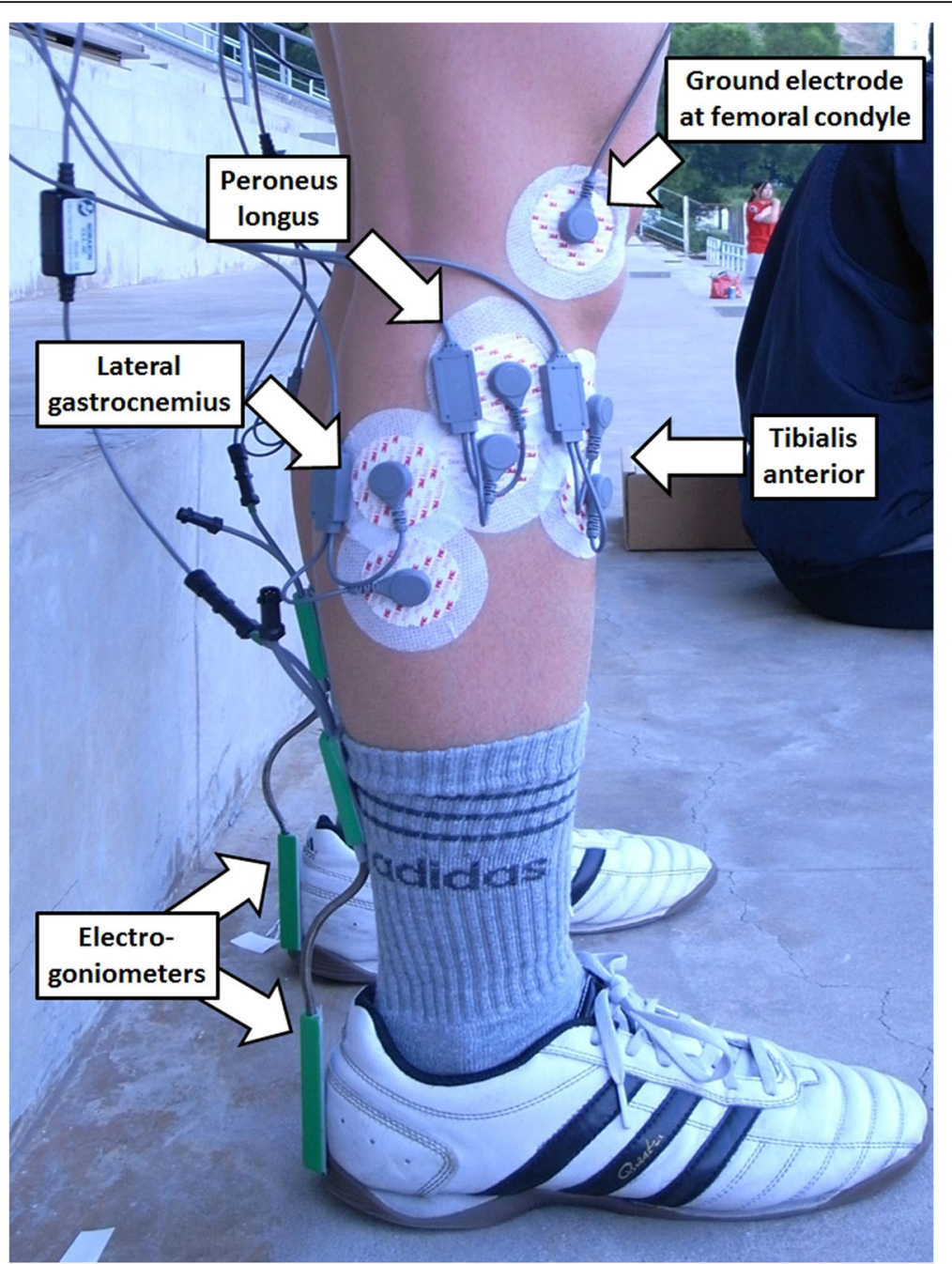

Fig. 2 The locations of electrodes for collecting electromyography signals, and the electrogoniometers for collection ankle inversion data

reaction time of each muscle. Each test took approximately $2 \mathrm{~min}$, and the subject resumed the exercise protocol immediately after. The average value of the reaction time from the three trials was used in the analysis.

\section{Statistical analysis}

Repeated measures one-way multivariate analysis of variance (MANOVA) was conducted on the dependent variables over time. If a significant time effect was found, a post-hoc Bonferroni test was conducted to evaluate if the reaction time at each time point significantly differed

Table 1 Demographic data of the participants $(N=7)$

\begin{tabular}{ll}
\hline Parameter & Mean \pm Standard Deviation \\
\hline Age & $25.7 \pm 1.3$ years \\
Height & $1.6 \pm 0.1 \mathrm{~m}$ \\
Body mass & $51.1 \pm 4.3 \mathrm{~kg}$ \\
\hline
\end{tabular}

from that at baseline. Statistical significance was set at $p<0.05$ level.

\section{Results}

Table 1 shows the demographic data of the participants. Table 2, Figs. 3 and 4 show the reaction time data for each of the three muscles for the dominant and nondominant limbs at each measurement point. MANOVA suggested a significant time effect for the muscle reaction time (Wilks' Lambda $=0.374, \mathrm{~F}=10.028, p=0.019$ ). Post-hoc tests indicated that the reaction times differed in both the dominant and non-dominant limbs compared with baseline. For the dominant limb, a longer reaction time was found after $15 \mathrm{~min}$ on the peroneus longus and tibialis anterior. For the non-dominant limb, a longer reaction time was also found after $15 \mathrm{~min}$ for all three muscles, and subsequently at $60 \mathrm{~min}$ on the tibialis anterior, at $75 \mathrm{~min}$ on the peroneus longus, and at 90 min on the lateral gastrocnemius. These longer reaction 
Table 2 Reaction times of each of the three muscles in the dominant and non-dominant limbs at different time points (standard deviation in bracket)

\begin{tabular}{|c|c|c|c|c|c|c|c|c|c|c|c|c|}
\hline \multirow[b]{3}{*}{ Time (min) } & \multicolumn{4}{|c|}{ Peroneus Longus } & \multicolumn{4}{|l|}{ Tibialis anterior } & \multicolumn{4}{|c|}{ Lateral gastrocnemius } \\
\hline & \multicolumn{2}{|l|}{ Dominant } & \multicolumn{2}{|l|}{ Non-dominant } & \multicolumn{2}{|l|}{ Dominant } & \multicolumn{2}{|l|}{ Non-dominant } & \multicolumn{2}{|l|}{ Dominant } & \multicolumn{2}{|l|}{ Non-dominant } \\
\hline & Reaction (ms) & Sig & Reaction (ms) & Sig & Reaction (ms) & Sig & Reaction (ms) & Sig & Reaction (ms) & Sig & Reaction (ms) & Sig \\
\hline 0 & $40.76(10.90)$ & - & $40.52(2.95)$ & - & $44.31(12.05)$ & - & $41.97(2.72)$ & - & $47.72(11.35)$ & - & $41.56(4.06)$ & - \\
\hline 15 & $47.98(7.32)$ & $.012^{*}$ & 48.97 (9.02) & $.043^{*}$ & $50.16(10.37)$ & $.004^{*}$ & $50.21(9.13)$ & $.030^{*}$ & $55.14(7.42)$ & .087 & $50.46(8.92)$ & $.037^{*}$ \\
\hline 30 & $49.07(3.00)$ & .083 & $42.11(8.86)$ & .701 & $50.58(3.88)$ & .222 & $42.27(9.10)$ & .941 & $53.01(4.64)$ & .313 & $42.62(9.36)$ & .833 \\
\hline 45 & $47.66(6.33)$ & .072 & $42.92(7.06)$ & .495 & $46.46(7.05)$ & .604 & $45.15(6.02)$ & .322 & $51.72(6.31)$ & .423 & $43.96(6.41)$ & .468 \\
\hline 60 & $48.08(7.20)$ & .239 & $44.40(6.23)$ & .162 & $49.98(8.04)$ & .373 & $48.01(7.20)$ & $.034^{*}$ & $50.77(7.93)$ & .598 & $45.38(5.16)$ & .253 \\
\hline 75 & $50.78(4.14)$ & .077 & $47.25(5.35)$ & $.001^{*}$ & $51.23(4.46)$ & .160 & $47.90(5.92)$ & $.006^{*}$ & $52.23(5.45)$ & .332 & $47.19(7.75)$ & .051 \\
\hline 90 & $49.31(5.98)$ & .196 & $45.74(4.00)$ & $.001^{*}$ & $50.78(6.55)$ & .328 & $47.93(6.15)$ & $.012^{*}$ & $52.94(7.02)$ & .387 & $47.77(4.31)$ & $.018^{*}$ \\
\hline 105 & $53.74(4.76)$ & $.049^{*}$ & $48.61(4.39)$ & $.003^{*}$ & $53.83(4.94)$ & .136 & $49.67(6.62)$ & $.017^{*}$ & $55.66(7.65)$ & .200 & $48.93(5.23)$ & $.010^{*}$ \\
\hline
\end{tabular}

times of the muscles of the non-dominant leg lasted until the end of the protocol (105 min).

\section{Discussion}

The results of the current study suggested that peroneal muscle fatigue may happen after the first 15 min of a simulated functional prolonged football protocol, and may cause delayed reaction time of the ankle muscles in this group of amateur female football players. A recent systematic video analysis reported that $68 \%$ of anterior cruciate ligament injuries happened in the first half, and one-quarter of them in the first $15 \mathrm{~min}$ of a football match [29]. This is probably due to a potentially higher injury risk caused by muscle fatigue [30] and subsequent reduction in postural control [31]. The authors suggested that this was possibly due to the inadequate neuromuscular readiness of fresh, unfatigued players [29]. Although that study was on another type of injury, the findings concur with the present study that muscle fatigue might already occur in the first $15 \mathrm{~min}$ of a match. This leads to delayed peroneal muscle times, as well as a greater risk of anterior cruciate ligament

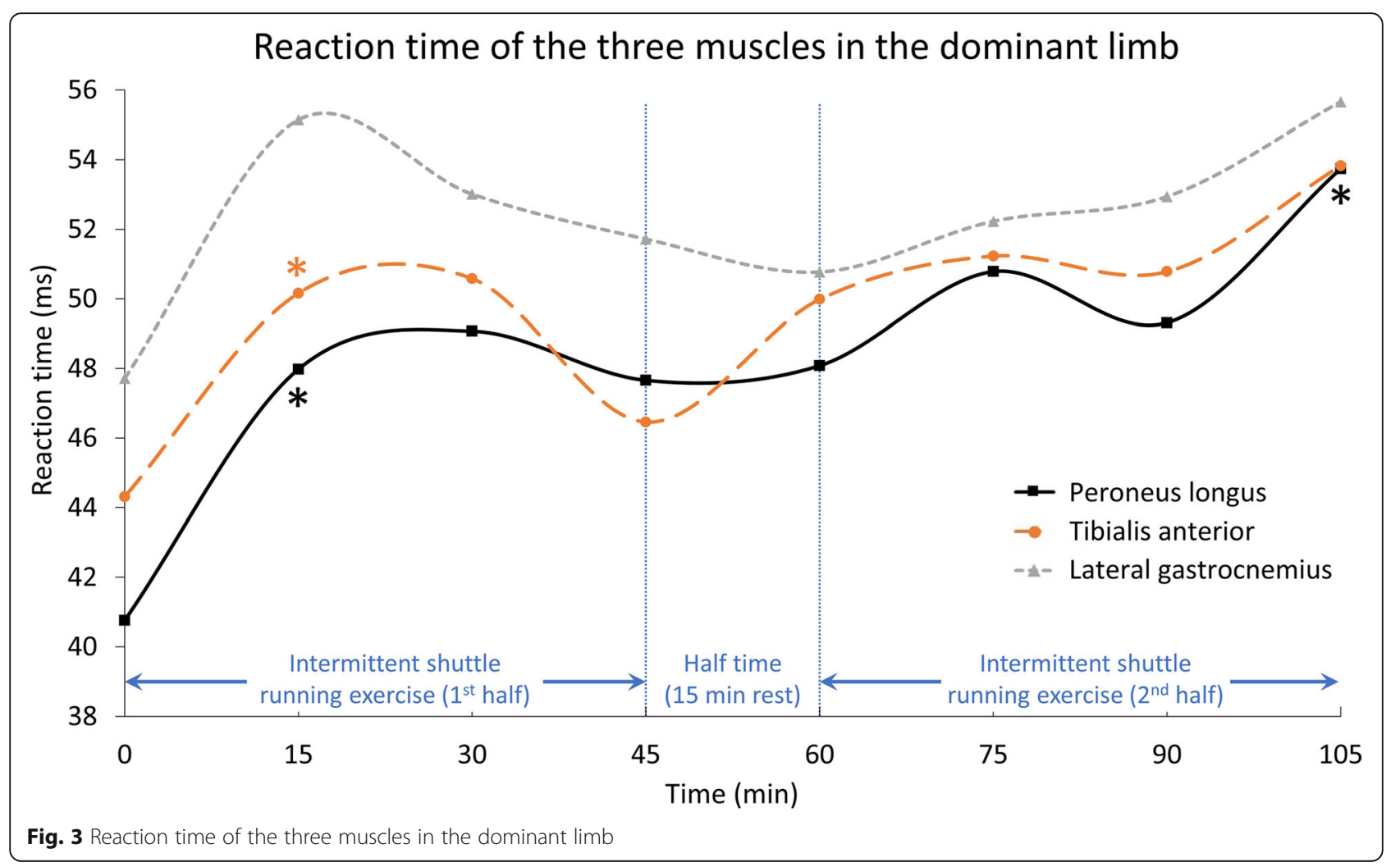




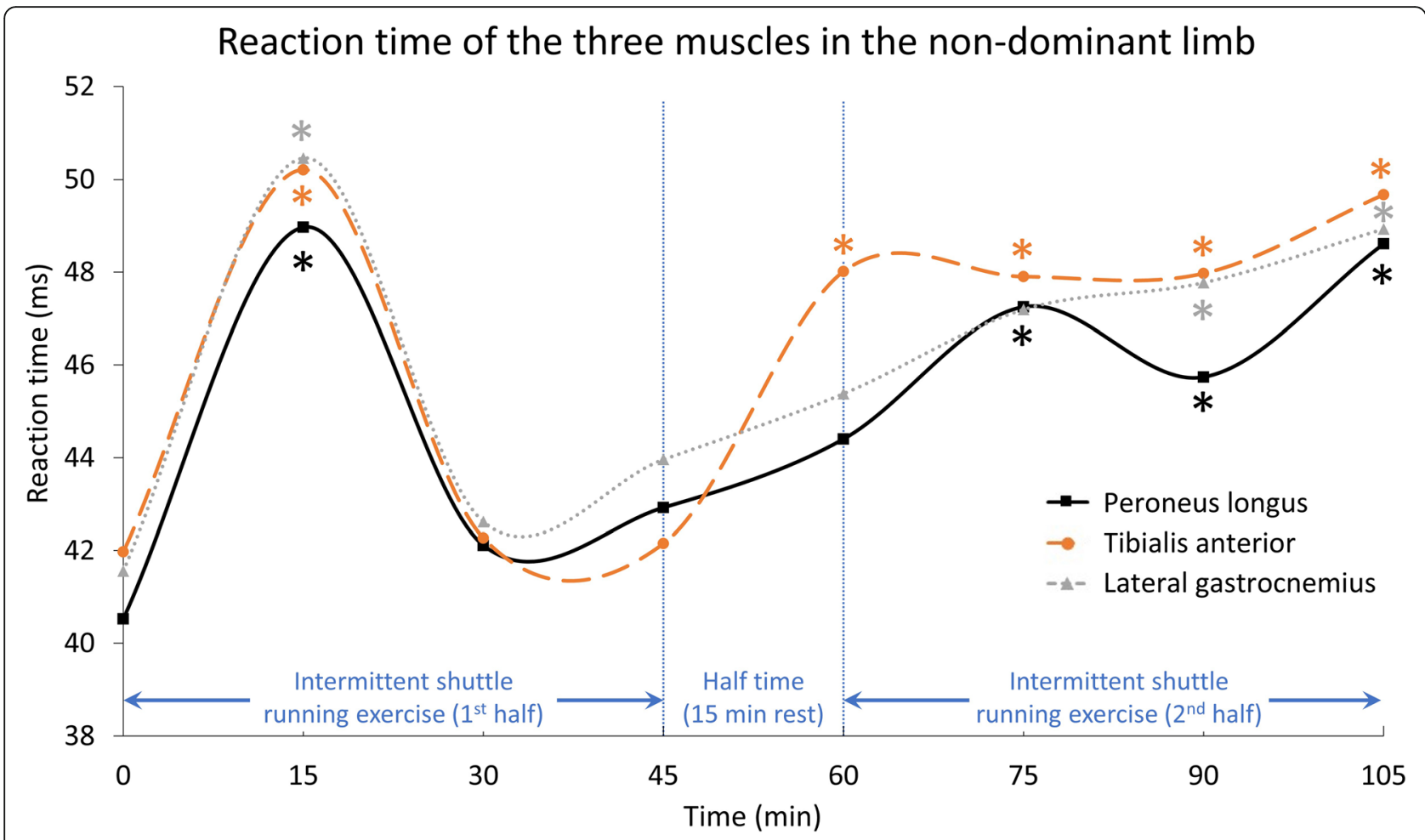

Fig. 4 Reaction time of the three muscles in the non-dominant limb

injuries. We observed that the reaction times plateaued after the first $15 \mathrm{~min}$ in the dominant limb (Fig. 3), before returning to nearer baseline at the 30th and 60th minutes. Subsequently, reaction times were slower again after the 75th minute, primarily in the non-dominant limb (Fig. 4). The reaction times of the studied muscles started from $40.5-47.7 \mathrm{~ms}$ and increased to $48.6-55.7$ $\mathrm{ms}$ at the end of the simulated protocol (105 min). Although these reaction times were still within the range found for healthy people, of 55-80 ms [32], the longer time in these athletes may be too great to react to a quick ankle sprain motion that happens within $50 \mathrm{~ms}$ [16]. The slower reaction time may thus impair functional ankle joint stability and result in more ankle injuries during the later stages of a football match [33].

Apart from the dominant lateral gastrocnemius, one interesting observation was the significantly increased reaction time in all muscles, from $40.5-44.3 \mathrm{~ms}$ to 48.0 $50.5 \mathrm{~ms}$, after the first $15 \mathrm{~min}$. The reaction time dropped back to $42.1-50.6 \mathrm{~ms}$ at $30 \mathrm{~min}$ but increased again at the end of the exercise protocol. Such acute delays in reaction time were also reported in a recent study with 10 male football players running for $45 \mathrm{~min}$ on a treadmill, and who had their reaction time to accommodate balance perturbation recorded every $7.5 \mathrm{~min}$ [34]. The reaction time in response to inversion perturbation, compared to that at the start $(63 \mathrm{~ms})$, did not differ at 7.5 and $15.0 \mathrm{~min}$, but raised significantly to $84-90 \mathrm{~ms}$ at
22.5, 30.0, and $37.5 \mathrm{~min}$, before returning to baseline, with no difference recorded at $45 \mathrm{~min}$. While the results of this study could not provide additional information to explain this finding, we believe that this acute delay in muscle reaction time may be a result of central fatigue, or central activation deficit, which has been demonstrated in prolonged running exercise in previous studies [35]. Unfortunately, to date, it is still very difficult to have a reliable quantitative method to investigate central fatigue. As discussed earlier, a recent study showed a quarter of anterior cruciate ligament injuries are recorded in the first $15 \mathrm{~min}$ of a football match [29]. This may imply that the readiness of a football player in term of muscle reaction and dynamic joint stability were not yet optimal after the conventional warm-up. Modification of warm-up protocol could be done by introducing new elements such as post-activation potentiation which was found to boost the performance of jumping and cutting movements in male footballers [36]. By introducing a warm-up exercise of moderate intensity (e.g. 80\%-1RM back squat) just before a football match, it might induce greater post-activation potentiation. This could improve the warm-up effect on the muscle activity and dynamic joint stability, but more research is needed.

Another interesting finding was the occurrence of delayed muscle reaction time in the non-dominant but not the dominant limb. De Luca and colleagues [37] conducted a myoelectric investigation on the fatigue at the 
first dorsal interosseous muscle and found that muscle fatigue happened faster in the non-dominant hand of right-handed individuals, but not in left-handed subjects. Farina and colleagues [38] also reported that the upper trapezius muscle at the non-dominant side was less fatigable in an isometric contraction test. To date, no studies have investigated the fatigue or reaction time of lower limb muscles during prolonged exercise.

There have been numerous attempts to deliver exercise training interventions to prevent inversion type ankle sprain injuries. Linford and colleagues [39] reported that a 6-week neuromuscular training programme was effective in reducing the reaction time of the peroneus longus muscles in a group of 26 healthy subjects. Similarly, Eils and Rosenbaum [40] reported that a 6-week multi-station proprioceptive exercise programme improved muscle reaction time, as well as joint position sense and postural sway. Interestingly, some other studies with proprioceptive and balance training as an intervention showed no reduction in muscle reaction time [41], but a significant effect in reducing ankle sprain incidents [42]. A previous study may give clues to this phenomenon. Sheth and colleagues [43] delivered an 8-week ankle disk training to 10 subjects and compared the contract time and pattern of four muscles - anterior tibialis, posterior tibialis, peroneus longus, and flexor digitorium longus. Although an expected decrease of reaction time did not happen, the contraction sequence changed to favour the correction of excessive ankle inversion. Before training, the four muscles tended to contract at the same time for a stiff ankle joint to accommodate excessive ankle inversion as initiated by a trapdoor platform. After training, the anterior and posterior tibialis contracted at a later time. These muscles are ankle invertors, so when they are inactive, they allow more eversion moment as generated by the ankle evertor, i.e. the peroneus longus. We believe that, if feasible, peroneal muscle endurance should be assessed in the preseason period [44], to understand the physical qualities and functional test performance of the football players and to design and guide injury prevention strategies.

\section{Conclusions}

In this study, a delayed reaction time of the ankle muscles was found after the first $15 \mathrm{~min}$ and towards the end of a simulated prolonged football protocol. The ankle muscle reaction time increased to values greater than the time taken for an ankle sprain motion $(<50$ ms). Such an increase may impair ankle joint stability, potentially resulting in more ankle injuries during the later stages of a football match. Acute delayed muscle reaction was observed in the first $15 \mathrm{~min}$, which may be a result of central fatigue or central activation deficit.
Future injury prevention strategies should also try to focus on tackling the delayed ankle muscle reaction time in the acute phase (the first $15 \mathrm{~min}$ ), in addition to the latter minutes in the second half.

\section{Acknowledgements \\ We acknowledge Dr. Stephen F. Burns of Nanyang Technological University Singapore for proofreading this article. \\ Authors' contributions \\ DTPF designed the study and was a major contributor in writing the manuscript. WCL collected, processed, analysed the data, and prepared the first draft of the manuscript. KMM managed the execution of the project. PSHY approved the study and facilitated the study with the necessary resources. All authors read and approved the final manuscript.}

\section{Funding}

The authors have not received funding for this study.

\section{Availability of data and materials}

The datasets used and/or analysed during the current study are available from the corresponding author on reasonable request.

\section{Ethics approval and consent to participate}

We have included a statement on ethics approval and consent, and the name of the ethics committee.

\section{Consent for publication}

Not applicable.

\section{Competing interests}

The authors declare that they have no competing interests.

\section{Author details}

${ }^{1}$ National Centre for Sport and Exercise Medicine, School of Sport, Exercise and Health Sciences, Loughborough University, Loughborough, UK.

${ }^{2}$ Department of Orthopaedics and Traumatology, Prince of Wales Hospital, Faculty of Medicine, The Chinese University of Hong Kong, Hong Kong, China. ${ }^{3}$ Sports Medicine Centre, Elite Training Science \& Technology Division, Hong Kong Sports Institute, Hong Kong, China. ${ }^{4}$ Student Services Centre, Lingnan University, Hong Kong, China.

Received: 8 June 2020 Accepted: 15 July 2020

Published online: 25 July 2020

\section{References}

1. Griffin J, Larsen B, Horan S, Keogh J, Dodd K, Andreatta M, Mihahan C (2020) Women's football: an examination of factors that influence movement patterns. J Strength Cond Res. https://doi.org/10.1519/JSC. 0000000000003638

2. Engstrom B, Johansson C, Tornkvist H (1991) Soccer injuries among elite female players. Am J Sports Med 19:372-375

3. Alamad TA, Kearney P, Cahalan R (2020) Injury in elite women's soccer: a systematic review. Phys Sportsmed. https://doi.org/10.1080/00913847.2020. 1720548

4. Mortvedt Al, Krosshaug T, Bahr R, Petushek E (2020) I spy with my little eye ... a knee about to go 'pop'? Can coaches and sports medicine professionals predict who is at greater risk of ACL rupture? Br J Sports Med 54:154-158

5. Prien A, Boudabous S, Junge A, Verhagen E, Delattre BMA, Tscholl PM (2020) Every second retired elite female football player has MRI evidence of knee osteoarthritis before age 50 years: a cross-sectional study of clinical and MRI outcomes. Knee Surg Sports Traumatol Arthrosc 28:353-362

6. Soligard T, Grindem H, Bahr R, Andersen TE (2010) Are skilled players at greater risk of injury in female youth football? Br J Sports Med 44:1118-1123

7. Sarmento H, Clemente FM, Marques A, Milanovic Z, Harper LD, Figueiredo A (2020) Recreational football is medicine against non-communicable diseases: a systematic review. Scand J Med Sci Sports 30:618-637

8. Wright IC, Neptune RR, van den Bogart AJ, Nigg BM (2000) The influence of foot positioning on ankle sprains. J Biomech 33:513-519 
9. Reilly T, Drust B, Clarke N (2008) Muscle fatigue during football match-play. Sports Med 38:357-367

10. Hoch MC, McKeon PO (2014) Peroneal reaction time after ankle sprain: a systematic review and meta-analysis. Med Sci Sports Exerc 46:546-556

11. Doherty C, Delahunt E, Caulfield B, Hertel J, Ryan J, Bleakley C (2014) The incidence and prevalence of ankle sprain injury: a systematic review and meta-analysis of prospective epidemiological studies. Sports Med 44:123-140

12. Fong DTP, Hong Y, Chan LK, Yung PWH, Chan KM (2007) A systematic review on ankle injury and ankle sprain in sports. Sports Med 37:73-94

13. Del Coso J, Herrero H, Salinero JJ (2018) Injuries in Spanish female soccer players. J Sport Health Sci 7:183-190

14. Larruskain J, Lekue JA, Diaz N, Odriozola A, Gil SM (2018) A comparison of injuries in elite male and female football players: a five-season prospective study. Scand J Med Sci Sports 28:237-245

15. Gaulrapp H, Becker A, Walther M, Hess H (2010) Injuries in women's soccer: a 1-year all players prospective field study of the women's Bundesliga (German premier league). Clin J Sport Med 20:264-271

16. Fong DTP, Hong Y, Shima Y, Krosshaug T, Yung PSH, Chan KM (2009) Biomechanics of supination ankle sprain: a case report of an accidental injury event in the laboratory. Am J Sports Med 37:822-827

17. Mok KM, Fong DTP, Krosshaug T, Engebretsen L, Hung ASL, Yung PSH, Chan KM (2011) Kinematics analysis of ankle inversion ligamentous sprain injuries in sports: 2 cases during the 2008 Beijing Olympics. Am J Sports Med 39: 1548-1552

18. Delahunt $\mathrm{E}$, Monaghan K, Caufield B (2007) Ankle function during hopping in subjects with functional instability of the ankle joint. Scand J Med Sci Sports 17:641-648

19. Patel NK, Murphy Cl, Pfeiffer TR, Naendrup JH, Zlotnicki JP, Debski RE, Hogan MV, Musahl V (2020) Sagittal instability with inversion is important to evaluate after syndesmosis injury and repair: a cadaveric robotic study. J Exp Orthop 7:18

20. de Noronha M, Lay EK, McPhee MR, Mnatzaganian G, Nunes GS (2019) Ankle sprain has higher occurrence during the latter parts of matches: systematic review with meta-analysis. J Sport Rehabil 28:373-380

21. Behan FP, Willis S, Pain MTG, Folland JP (2018) Effects of football simulated fatigue on neuromuscular function and whole-body response to disturbances in balance. Scand J Med Sci Sports 28:2547-2557

22. Gribble PA, Hertal J, Denegar CR (2007) Chronic ankle instability and fatique create proximal joint alterations during performance of the star excursion balance test. Int J Sports Med 28:236-242

23. Jackson ND, Gutierrez GM, Kaminski T (2009) The effect of fatigue and habituation on the stretch reflex of the ankle musculature. J Electromyogr Kinesiol 19:75-84

24. Rahnama N, Lees A, Reilly T (2006) Electromyography of selected lower-limb muscles fatigued by exercise at the intensity of soccer match-play. J Electromyogr Kinesiol 16:257-263

25. Karlsson J, Andreasson GO (1992) The effect of external ankle support in chronic lateral ankle joint instability: an electromyographic study. Am J Sports Med 20:257-261

26. Nicholas CW, Nuttall FE, Williams C (2000) The Loughborough intermittent shuttle test: a field test that simulates the activity pattern of soccer. J Sports Sci 18:97-104

27. Chan YY, Fong DTP, Yung PSH, Fung KY, Chan KM (2008) A mechanical supination sprain simulator for studying ankle supination sprain kinematics. J Biomech 41:2571-2574

28. Perotto AO (2005) Anatomical guide for the electromyographer: the limb and trunk, 4th edn. Charles C Thomas Publisher Ltd, Springfield, pp 175-192

29. Della Villa F, Buckthorpe M, Grassi A, Nabiuzzi A, Tosarelli F, Zaffagnini S, Della Villa S (2020) Systematic video analysis of ACL injuries in professional male football (soccer): injury mechanisms, situational patterns and biomechanics study on 134 consecutive cases. Br I Sports Med. https://doi. org/10.1136/bjsports-2019-101247

30. Almonroeder TG, Tighe SM, Miller TM, Lanning CR (2020) The influence of fatigue on decision-making in athletes: a systematic review. Sports Biomech 19:76-89

31. Valldecabres R, Richards J, De Benito AM (2020) The effect of match fatique in elite badminton players using plantar pressure measurements and the implications to injury mechanisms. Sports Biomech. https://doi.org/10.1080/ 14763141.2020.1712469

32. Konradsen L, Ravn JB (1991) Prolonged peroneal reaction time in ankle instability. Int J Sports Med 12:290-292
33. Greig M, Walker-Johnson C (2007) The influence of soccer-specific fatigue on functional stability. Phys Ther Sport 8:185-190

34. Lohkamp M, Craven S, Walker-Johnson C, Greig M (2009) The influence of ankle taping on changes in postural stability during soccer-specific activity. J Sport Rehabil 18:482-492

35. Millet GY, Lepers R (2004) Alterations of neuromuscular function after prolonged running, cycling and skiing exercises. Sports Med 34:105-116

36. Petisco C, Ramirez-Campillo R, Hernández D, Gonzalo-Skok O, Nakamura FY, Sanchez-Sanchez J (2019) Post-activation potentiation: effects of different conditioning intensities on measures of physical fitness in male young professional soccer players. Front Psychol 10:1167

37. De Luca CJ, Sabbahi MA, Roy SH (1986) Median frequency of the myoelectric signal: effects of hand dominance. Eur J Appl Physiol 55:457-464

38. Farina D, Kallenberg LA, Merletti R, Hermens HJ (2003) Effect of side dominance on myoelectric manifestations of muscle fatique in the human upper trapezius muscle. Eur J Appl Physiol 90:480-488

39. Linford CW, Hopkins JT, Schulthies SS, Freland B, Draper DO, Hunter I (2006) Effects of neuromuscular training on the reaction time and electromechanical delay of the peroneus longus muscle. Arch Phys Med Rehabil 87:395-401

40. Eils E, Rosenbaum D (2001) A multi-station proprioceptive exercise program in patients with ankle instability. Med Sci Sports Exerc 33:1991-1998

41. Hertel J (2000) Functional instability following lateral ankle sprain. Sports Med 29:361-371

42. Verhagen E, van der Beek A, Twisk J, Bouter L, van Mechelen W (2004) The effect of a proprioceptive balance board training program for the prevention of ankle sprains: a prospective controlled trial. Am J Sports Med 32:1385-1393

43. Sheth P, Yu B, Laskowski ER, An KN (1997) Ankle disk training influences reaction times of selected muscles in a simulated ankle sprain. Am J Sports Med 25:538-543

44. Delvaux F, Schwartz C, Rodriguez C, Forthomme B, Kaux JF, Croisier JL (2020) Preseason assessment of anaerobic performance in elite soccer players: comparison of isokinetic and functional test. Sports Biomech. https://doi.org/10.1080/14763141.2020.1750681

\section{Publisher's Note}

Springer Nature remains neutral with regard to jurisdictional claims in published maps and institutional affiliations.

\section{Submit your manuscript to a SpringerOpen ${ }^{\circ}$ journal and benefit from:}

- Convenient online submission

- Rigorous peer review

- Open access: articles freely available online

High visibility within the field

- Retaining the copyright to your article

Submit your next manuscript at $\boldsymbol{\nabla}$ springeropen.com 